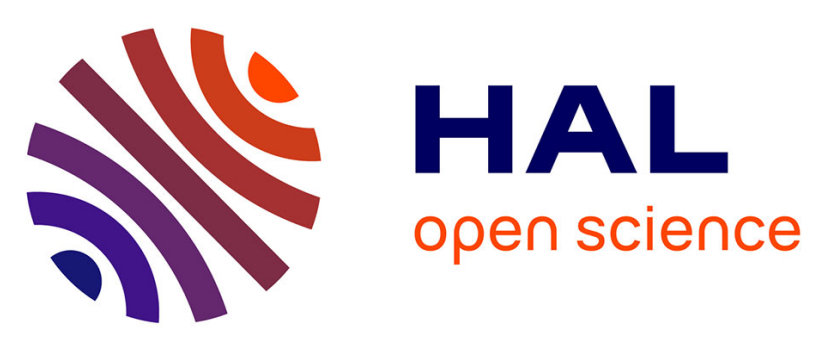

\title{
Fonctionnement cognitivo-émotionnel : le rôle de l'intensité émotionnelle chez les individus anxieux
}

Aurélie Pasquier, Agnès Bonnet, Jean-Louis Pedinielli

\section{To cite this version:}

Aurélie Pasquier, Agnès Bonnet, Jean-Louis Pedinielli. Fonctionnement cognitivo-émotionnel : le rôle de l'intensité émotionnelle chez les individus anxieux. Annales Médico-Psychologiques, Revue Psychiatrique, 2009, 167 (9), pp.649-656. 10.1016/j.amp.2007.11.017 . hal-01271880

\section{HAL Id: hal-01271880 \\ https://hal-amu.archives-ouvertes.fr/hal-01271880}

Submitted on 9 Feb 2016

HAL is a multi-disciplinary open access archive for the deposit and dissemination of scientific research documents, whether they are published or not. The documents may come from teaching and research institutions in France or abroad, or from public or private research centers.
L'archive ouverte pluridisciplinaire HAL, est destinée au dépôt et à la diffusion de documents scientifiques de niveau recherche, publiés ou non, émanant des établissements d'enseignement et de recherche français ou étrangers, des laboratoires publics ou privés. 


\title{
Fonctionnement cognitivo-émotionnel : Le rôle de l'intensité émotionnelle chez les individus anxieux
}

\author{
Aurélie Pasquier (1), Agnès Bonnet (2), Jean-Louis Pedinielli (3) \\ 1 Doctorante, Attaché Temporaire d'Enseignement et de Recherche* ** \\ 2 Maître de Conférence en Psychologie* \\ 3 Professeur de Psychopathologie*
}

*Centre de recherches en Psychologie de la Connaissance, du Langage et de l'Emotion (PsyCLE, EA3273).

Axe thématique III « Emotions, Construction du réel et Pathologie ».

Université de Provence

21, avenue Robert Schuman

13621 Aix-en-Provence Cedex 1

**Département Adaptation Scolaire et Scolarisation des élèves Handicapés (ASH)

Université de Provence

Institut Universitaire de Formation des Maîtres d'Aix-Marseille I

2, avenue Jules Isaac

13626 Aix-en-Provence Cedex 1

Article publié dans les Annales Médico-Psychologiques en novembre 2009 (vol.167 n9, p.641-716). 


\title{
Fonctionnement cognitivo-émotionnel : Le rôle de l'intensité émotionnelle chez les individus anxieux
}

\begin{abstract}
Résumé
L'anxiété est un trouble émotionnel très répandu dans la population générale et qui semble être souvent en lien avec les symptômes dépressifs. L'objectif de cette étude est de tester l'hypothèse de relations entre fonctionnement cognitivo-émotionnel, symptômes anxieux et dépressifs. Un groupe de 50 sujets âgés de 18 à 65 ans constitue l'échantillon de notre étude. Les participants ont rempli l'échelle d'Anxiété et de Dépression (HAD), les échelles d'intensité et d'expressivité émotionnelle (AIM et EES) et l'échelle de niveau de conscience émotionnelle (LEAS). Les résultats montrent l'existence de corrélations positives significatives entre les symptômes anxieux, l'intensité émotionnelle, l'expressivité émotionnelle et le niveau de conscience émotionnelle. Ces résultats soulignent l'importance des variables émotionnelles dans l'étude et la prise en charge des individus anxieux.
\end{abstract}

Mots-clés : Anxiété ; Conscience émotionnelle; Expressivité émotionnelle ; Intensité émotionnelle.

\section{Cognitive-emotional functioning: Role of emotional intensity in the anxiety}

\begin{abstract}
Summary :
Anxiety is an emotional trouble very present in the general population and it seems to be linked to depression symptoms. The aim of this study is to test the relations hypothesis between cognitive-emotional functioning, anxiety and depressive symptoms. The study's sample was formed from a group of 50 subjects, aged from 18 to 65. Participants completed Hospital Anxiety and Depression Scale (HAD), Affect Intensity Measure (AIM), Emotional Expressivity Scale (EES) and Levels of Emotional Awareness Scale (LEAS). Our results show strong positive correlations between anxiety symptoms, affect intensity, emotional expressivity and level of emotional awareness. In conclusion, this results point out the importance of emotional variables in the study and treatment of subjects suffering of anxiety symptoms.
\end{abstract}

Key-words : Anxiety ; Affect intensity ; Emotional Awareness ; Emotional expressivity. 


\section{Introduction}

Les états anxieux constituent actuellement un véritable problème de santé publique. Une étude française en médecine ambulatoire [7] rapporte que l'anxiété est un état répandu dans notre société et qui plus est représenterait un facteur de risque de la dépression et des conduites addictives de type consommation d'alcool ou de médicaments [3]. De nombreux travaux mettent en évidence la présence de facteurs émotionnels pouvant expliquer des symptômes psychopathologiques tels que l'anxiété.

\subsection{Définition de l'anxiété}

«L'anxiété est un état émotionnel fait sur le plan phénoménologique de trois éléments fondamentaux : la perception d'un danger imminent, une attitude d'attente devant ce danger et un sentiment de désorganisation lié à la conscience d'une impuissance totale en face de ce danger » [25] (p.32). D'un point de vue clinique, l'anxiété peut être considérée comme un signe des états dépressifs [24]. En effet, le clinicien retrouve souvent dans sa pratique la coexistence de symptômes anxieux et dépressifs sans que les uns et les autres ne soient totalement présents pour parler de troubles anxieux et dépressifs. C'est pourquoi dans cette recherche, nous avons fait le choix d'aborder ces symptomatologies selon une approche dimensionnelle pour rester au plus près de nos observations cliniques plutôt que d'adopter une approche psychopathologique visant à établir des catégories. Ainsi, l'anxiété peut aussi être considérée comme une émotion qui renvoie à un état émotionnel faisant lui-même partie d'un fonctionnement émotionnel plus global. Ce dernier se définit à l'aide de dimensions cognitivo-émotionnelles qui structurent l'expérience émotionnelle de l'individu. 


\subsection{Le fonctionnement émotionnel}

Le fonctionnement émotionnel d'un individu est composé de dimensions qui structurent son expérience émotionnelle. Cette structure comporterait deux dimensions indépendantes : la «valence » de l'émotion et son «activation »[31]. La première dimension correspond à la manière «agréable ou désagréable » dont les individus vivent les expériences subjectives [26]. La deuxième dimension correspond à la force avec laquelle une émotion est vécue par un individu [20] [10] [11]. Pour certains les deux dimensions émotionnelles peuvent être croisées [5]. Des études ont montré que les dimensions de valence et d'activation de la structure émotionnelle pouvaient être en lien avec des symptômes anxieux et dépressifs [30]. D'autres travaux ont cherché à mettre en évidence les relations qui existent entre les capacités de différenciation et de description de ses propres émotions et celles d'autrui, l'affectivité positive et les symptômes anxieux [6]. Les capacités de différenciation et de description des émotions correspondent à un niveau de développement structurel de la conscience émotionnelle de l'individu appelé «niveau de développement cognitivo-émotionnel» [16]. L’appréhension de cette dimension peut fournir des éléments de compréhension de la symptomatologie anxieuse. Ceci dans la mesure où les symptômes anxieux seraient associés à un faible niveau de développement de la conscience émotionnelle [6]. Celui-ci pourrait jouer un rôle étiologique dans la symptomatologie anxieuse, d'autant qu'un rapprochement a été fait entre les stades cognitivo-émotionnels les plus «précaires» et l'alexithymie (terme désignant «absence de mots pour décrire ses émotions » proposé par Sifnéos 
[27]), envisagée alors comme trait de personnalité. De plus on connât les liens qu'entretiennent la symptomatologie anxieuse et l'alextihymie [22].

\subsection{Objectifs}

Dans cette recherche, nous suivons quatre objectifs majeurs : le premier est de montrer les relations pouvant exister entre les symptomatologies anxieuse et dépressive et les tendances à ressentir les émotions avec intensité (IE) et à se considérer comme expressif d'un point de vue émotionnel (EE). Le deuxième objectif est d'analyser les relations entre niveau de conscience émotionnelle (dans les trois formes de conscience émotionnelle globale, intra et inter-subjective, notées CEG, CE-Intra et CE-Inter) et les symptômes anxieux et dépressifs. Le troisième est de déterminer s'il existe des différences sur l'ensemble de ces composantes (IE, EE, CEG, CE-Intra et CE-Inter) selon les groupes d'âge des individus considérés. Enfin, le quatrième objectif est de proposer, par l'analyse factorielle des échelles utilisées, une étude des liens pouvant exister entre les différentes dimensions des échelles. Ceci peut permettre de dégager des dynamiques de fonctionnement émotionnel qui varient en fonction de la présence ou de l'absence de symptômes anxieux et dépressifs.

\section{Hypothèses}

Pour rendre compte des relations existant entre symptômes anxieux et dépressifs, dimensions émotionnelles et niveau de conscience émotionnelle, nous avons formulé quatre hypothèses opératoires :

- Il existe des corrélations significatives entre le niveau d'anxiété, l'intensité émotionnelle et le niveau de conscience émotionnelle ; 
- Il existe des corrélations négatives entre les symptomatologies anxieuse et dépressive et les niveaux de conscience émotionnelle (sur les trois formes CEG, CE-Intra et CE-Inter) ;

- Les dimensions émotionnelles (IE et $\mathrm{EE}$ ) et le niveau de conscience émotionnelle varient selon l'âge des individus ;

- Il existe des profils de fonctionnement émotionnel qui diffèrent selon la présence ou l'absence de symptômes anxieux et dépressifs.

\section{Méthodologie}

\subsection{Participants}

Notre échantillon est constitué de 50 femmes âgées de 18 à 65 ans appariés en âge. L’âge moyen est de 43.3 ans avec un écart type de 13.47 ans. La moitié des participants $(\mathrm{N}=25)$ présente un état anxieux pathologique et un état dépressif non pathologique. Elles ont été recrutées dans des cabinets de médecins généralistes selon des critères de symptomatologie anxieuse (critères DSM-IV-TR), repérés par le médecin. Malgré leur recrutement dans le cadre d'une consultation médicale, il s'agit de patientes qui ne prennent pas de traitements médicamenteux de type anxiolytique ou anti-dépresseur. Les participantes restantes $(\mathrm{N}=25)$ ne présentent ni d'état anxieux ni d'état dépressif pathologique et sont issues de la population tout-venante.

\subsection{Les instruments}

\subsubsection{Mesure de l'anxiété}

Le «Hospital Anxiety Depression» (HAD) est un auto-questionnaire structuré en 14 items. Il évalue le niveau actuel de la symptomatologie anxieuse et dépressive [32]. Cet outil a été validé en France par Lépine et collaborateurs [21]. Un score est attribué en anxiété et en dépression en 
fonction des réponses choisies. Nous nous sommes référés à la cotation de Barczack et al. [1] selon laquelle une note de 8 et plus constituerait le seuil optimal avec pour l'anxiété et la dépression respectivement des sensibilités de 82 et $70 \%$ et des spécificités de 94 et $68 \%$.

\subsubsection{Mesures des dimensions émotionnelles}

Nous avons choisi d'évaluer l'intensité émotionnelle au moyen de l' «Affect Intensity Measure » (AIM). Il s'agit d'un auto-questionnaire en 40 items développé par Larsen [19]. Il permet d'évaluer la force avec laquelle une émotion est ressentie. Cette conception unidimensionnelle de Larsen a été revue et corrigée par Bryant et al. [5].

L’ «Emotional Expressivity Scale » (EES) est un auto-questionnaire en 17 items qui cherche à évaluer la façon dont les individus perçoivent leur expressivité (physiologique, verbale et non-verbale) [14]. Cette échelle fait encore l'objet d'études de validation en France.

\subsubsection{Mesure du niveau de développement cognitivo-émotionnel}

Nous avons choisi d'utiliser l'Echelle de Niveaux de Conscience Emotionnelle (Level of Emotional Awareness Scale ou LEAS) développée par Lane et Schwartz [16]. Il s'agit d'un auto-questionnaire composé de 20 situations dans lesquelles on demande aux sujets de se représenter dans une courte scène le mettant en interaction avec une autre personne. Les réponses sont cotées de 0 à 5 et permettent d'obtenir un score global de 0 à 100 avec deux sous-scores évaluant les niveaux de conscience émotionnelle intra et inter-subjectif. 


\subsection{Procédure}

La passation était individuelle. Toutes les tâches étaient présentées dans un ordre fixe (AIM_EES_LEAS_HAD) pour écarter les effets d'ordre de présentation des questionnaires selon les participants, et sans limite de temps. L'outil «HAD» nous a permis de différencier nos sujets sur les scores d'anxiété et de dépression. Sont considérés comme présentant une symptomatologie anxieuse pathologique et une symptomatologie dépressive considérée comme non pathologique («état anxieux pathologique » et état dépressif non pathologique), ceux qui ont obtenu une note supérieure à huit pour chaque partie évaluant l'anxiété et la dépression. Nous avons fait le choix de ne pas utiliser ces valeurs seuil comme un critère de constitution de groupes de sujets. Nous avons opté pour une approche dimensionnelle de la symptomatologie anxieuse principalement, tout en prenant en compte les données se rapportant à la symptomatologie dépressive. Les sujets de cette étude se distribuent sur un continuum anxieux autour d'une valeur centrale qu'est la valeur seuil de la symptomatologie anxieuse considérée comme pathologique (état anxieux pathologique). Quant aux scores de dépression, aucun ne dépasse la valeur seuil de la symptomatologie dépressive pathologique (état dépressif non pathologique).

\subsection{Analyses statistiques}

Les analyses statistiques descriptives, inférentielles (corrélations), nonparamétriques (test de «Kruskal-Wallis ») et factorielles (test en Composantes Principales) ont toute été réalisées à l'aide du logiciel Statwiew dans sa version 5.0 et les représentations graphiques et les tableaux ont été faits avec le logiciel Excel 5.0. 


\section{Résultats}

\subsection{Analyses descriptives}

Les scores aux différentes échelles ainsi qu'aux sous-échelles ont été calculés. Notons que pour l'ensemble des échelles utilisées, un score élevé aux mesures correspond à une position élevée sur la dimension mesurée. Le tableau 1 présente l'analyse descriptive (moyenne, écart-type, médiane, minimum, maximum) des différentes mesures d'état (anxieux et dépressif), de dimensions émotionnelles (facteur global d' «intensité émotionnelle »(IEG) et les sousfacteurs «d'affectivité positive» (AP), «intensité négative» (IN) et «réactivité négative» $(\mathrm{RN})$ ainsi que le facteur global d'«expressivité émotionnelle » $(\mathrm{EE}))$ et de niveau de développement cognitivo-émotionnel (CEG, CE-Intra et CE-Inter).

\section{$\underline{\text { Tableau } 1}$}

Le questionnaire HAD permet d'obtenir deux mesures du type «symptômes anxieux » et «symptômes dépressifs ». On peut observer que la moyenne de l'ensemble des sujets $($ moy $=7,92)$ sur la variable mesurée « symptômes anxieux » est proche de la valeur seuil (score $=8$ ) avec des notes minimale à 2 et maximale à $15(\mathrm{t}=20,85 ; \mathrm{p}<0,0001)$. De plus, la médiane est à 7,50 ce qui signifie que l'effectif global est constitué de sujets qui se distribuent de manière homogène sur un continuum allant de la symptomatologie anxieuse à la symptomatologie anxieuse pathologique autour d'une médiane qui correspond à la note seuil du caractère pathologique des symptômes anxieux. Le tableau 1 nous renseigne aussi sur l'absence de symptomatologie dépressive pathologique (la note maximale est égale à 7 , ce qui est inférieur à la valeur seuil pour déterminer le caractère pathologique des 
symptômes dépressifs qui est de 8) au sein de notre effectif global que les sujets souffrent d'anxiété pathologique ou non $(t=12,42 ; \mathrm{p}<0,0001)$.

\subsection{Corrélations}

Nous pouvons observer qu'il existe des corrélations significatives entre symptomatologies anxieuse et dépressive, dimensions émotionnelles et niveau de développement cognitivo-émotionnel pour l'ensemble des sujets (pathologiques/tout-venants).

4.2.1. Relation entre âge, symptômes, dimensions émotionnelles et développement cognitivo-émotionnelle

Il existe de nombreuses relations significatives entre l'âge, les symptômes dépressifs, l'intensité négative, l'expressivité émotionnelle et les niveaux de conscience émotionnelle globale, intra et inter-subjective. On constate la présence d'une corrélation positive significative entre l'âge et la dépression. Plus l'âge du sujet augmente et plus les symptômes dépressifs sont présents. Il existe une corrélation modérée entre l'âge et l'intensité négative, ce qui signifie que plus le sujet avance en âge et plus il présente une tendance à ressentir les émotions négatives de façon intense. On note aussi la présence de corrélations significatives négatives entre l'âge et l'expressivité émotionnelle. Plus l'âge augmente, moins le sujet se considère comme expressif sur le plan émotionnel. De même, l'âge est négativement corrélé à la conscience émotionnelle globale ainsi qu'aux deux sous-facteurs de conscience émotionnelle intra- et inter- subjectives. En d'autres termes, plus on est âgé et moins l'on est capable de différencier et de décrire ses états émotionnels et ceux d'autrui.

$\underline{\text { Tableau } 2}$ 


\subsubsection{Relation entre anxiété, dépression, intensité émotionnelle}

et conscience émotionnelle

Il existe une corrélation significative positive entre les symptômes anxieux et les symptômes dépressifs. Cela signifie que lorsque la symptomatologie anxieuse augmente, celle de la dépression s'accroît aussi. On remarque aussi des corrélations significatives positives entre les symptômes anxieux et l'intensité émotionnelle et ses trois sous-facteurs (AP, IN et RN). Plus les individus sont anxieux et plus ils ont tendance à ressentir les émotions avec intensité qu'elles soient positives comme négatives. Il existe des corrélations positives entre la symptomatologie anxieuse et la conscience émotionnelle ainsi qu'avec les deux sous-facteurs (CE-Intra et CE-Inter). Cela signifie que plus l'anxiété augmente et plus l'individu est capable d'identifier et de décrire ses émotions et celles des autres.

\subsubsection{Relation entre dépression, intensité, expressivité et conscience émotionnelle}

On note la présence de corrélations positives entre les symptômes dépressifs et l'intensité émotionnelle négative, ainsi qu'avec la réactivité négative. En d'autres termes plus la dépression augmente et plus l'individu ressent et réagit aux stimuli émotionnels de façon intense et négative. De plus, on remarque l'existence d'une corrélation négative entre la symptomatologie dépressive et l'expressivité émotionnelle. Cela signifie que plus on est en proie à une humeur dépressive et moins on se considère comme expressif sur le plan émotionnel. On remarque aussi que l'intensité émotionnelle est corrélée positivement avec le niveau de conscience émotionnelle intra-subjectif et que la réactivité négative est corrélée positivement à la conscience émotionnelle 
globale et intra-subjective. En d'autres termes, plus les individus ont un vécu émotionnel intense et réagissent de manière négative aux stimuli émotionnels et plus ils parviennent à différencier et décrire leurs propres états émotionnels.

\subsection{Etude de l'effet de l'âge}

Nous observons des différences de moyennes significatives entre les quatre groupes d'âge constitués (groupe 1 «19-29 ans », groupe 2 «32-39 ans », groupe 3 «42-49 ans » et groupe 4 «53-65 ans ») pour certaines dimensions cognitivo-émotionnelles. Nous observons des différences de moyennes significatives pour les facteurs d'«expressivité émotionnelle», de « conscience émotionnelle globale » et de «niveau de conscience émotionnelle intra-subjectif ».

\section{Diagramme 1}

Les personnes se situant entre «35-45 ans », suivies de près par les «45-55 ans », se perçoivent comme étant les plus expressives d'un point de vue émotionnel par rapport aux plus jeunes «19-29 ans » puis aux plus âgés «53-65 ans ». Les personnes les plus jeunes de notre échantillon se placent à un niveau de développement de conscience des émotions plus élevé par rapport aux autres individus (notamment les 45-55 ans). Les plus jeunes ont aussi un niveau de conscience émotionnelle intra-subjectif plus élevé que les autres personnes comparées (notamment les plus âgées « 53-65 » ans). Concernant les autres dimensions émotionnelles évaluées, les sujets ne différent pas selon leur groupe d'âge.

\subsection{Analyse factorielle}

Nous avons mené une analyse factorielle de type «Composante Principale » pour l'ensemble des sujets $(\mathrm{N}=50)$ afin de mettre en évidence 
l'existence de liens entre plusieurs dimensions sous-jacentes aux échelles utilisées. L'étude des valeurs propres ainsi que l'analyse des parts de variance expliquées par les axes factoriels montre l'importance de trois facteurs dans la compréhension de la symptomatologie anxieuse associée ou non à des symptômes dépressifs.

\section{$\underline{\text { Tableau } 3}$}

Un premier facteur comporte les variables «symptômes anxieux», «intensité émotionnelle», «affectivité positive», «intensité négative », «réactivité négative», «expressivité émotionnelle» et «conscience émotionnelle globale» et «conscience émotionnelle intra-subjective». Elles constituent un axe factoriel que nous identifions comme la dimension « connaissance de soi ». Ce premier facteur est appelé ainsi dans la mesure où il regroupe les dimensions émotionnelles et le niveau de développement cognitivo-émotionnel qui renvoient à la façon dont les individus se considèrent sur des dimensions d'anxiété, d'intensité et d'expressivité émotionnelle, ainsi que sur des niveaux développementaux de différenciation et de descriptions des états émotionnels propres. La conscience émotionnelle ferait partie d'un même ensemble de capacités où co-existent les représentations de soi concernant nos façons de réagir aux stimuli émotionnels. Il semble alors que les symptômes anxieux soit en lien avec le fait de se considérer comme ressentant les émotions positives et négatives de manière plutôt intense, et comme étant expressif d'un point de vue émotionnel. Enfin, la symptomatologie anxieuse serait en lien avec des capacités d'identification et de description de ses propres émotions.

Un deuxième facteur est composé des variables «conscience émotionnelle globale », niveaux de conscience intra-subjectif et inter-subjectif, 
qui ont des saturations élevées dès lors que l'âge obtient une valeur significative négative et que l'anxiété et la dépression n'apparaissent plus de manière significative. Il s'agit des capacités de l'individu identifier et à attribuer des émotions à soi-même et à autrui. Nous avons identifié ce facteur comme étant la dimension de «différenciation des états émotionnels ». Cette dénomination renvoie au fait que sur cette dimension, les sujets sont capables de procéder à une description fine de leurs différents états émotionnels. Ceci est différent du précédent facteur dans la mesure où la perception de l'intensité émotionnelle n'intervenant plus, une identification et description plus complexe de ses propres émotions et de celles d'autrui est possible. On peut repérer ceci à partir des valeurs négatives proches de la significativité présentes au sein du deuxième axe factoriel (intensité émotionnelle et intensité négative). Les capacités de différenciation émotionnelle fine entretiendraient des relations inverses avec l'âge, les dimensions d'intensité et d'expressivité émotionnelles (ont des coordonnées factorielles négatives). C'est pourquoi l'anxiété n'apparaîtrait plus comme ayant un poids dans cet axe factoriel (poids factoriel de l'anxiété égal à 0,02 ).

Enfin, les symptômes anxieux et dépressifs ainsi que l'expressivité émotionnelle ont des saturations élevées sur le troisième axe et forment le troisième facteur. Les symptomatologies anxieuse et dépressive ont des coordonnées factorielles positives alors que l'expressivité émotionnelle a des coordonnées factorielles négatives. Cet axe factoriel renvoie à la dimension d' «abrasion émotionnelle ». Celle-ci se rapporte à l'idée que les sujet anxieux se perçoivent comme non expressif sur le plan émotionnel lorsque les symptômes dépressifs sont présents, ce qui indique que l'individu est alors dominé par des 
émotions négatives qui viennent comme «abraser » son vécu émotionnel et ses capacités de réponse aux stimuli émotionnels. Les symptômes dépressifs associés à ceux d'anxiété agiraient sur les capacités de représentations de l'individu concernant sa propre expressivité émotionnelle. De ce fait, tous les estimateurs de cette représentation de l'expressivité émotionnelle (intensité émotionnelle globale et spécifique) ne pèsent plus sur ce troisième axe. Quant au niveau de conscience émotionnelle, lorsque l'anxiété est associée à la dépression, il n'a plus de poids significatif. Les symptomatologies dépressive et anxieuse viendraient diminuer les capacités du sujet à différencier et décrire ses propres états émotionnels et ceux d'autrui.

Chacun des facteurs a une valeur propre supérieure à 1 (règle de Kaiser). Les facteurs « connaissance de soi », « niveau de différenciation des états émotionnels » et «abrasion émotionnelle » expliquent à eux trois plus de $75 \%$ de la variance totale des sujets.

\section{Discussion}

Nos résultats indiquent qu'il existe des différences sur les dimensions émotionnelles et le niveau de développement cognitivo-émotionnel selon la symptomatologie des individus.

Notre première hypothèse concernant l'existence de corrélations significatives entre la symptomatologie anxieuse et l'intensité émotionnelle (IE) est validée. Il apparaît en effet que les symptômes anxieux sont significativement corrélés avec la dimension émotionnelle IE. La deuxième hypothèse sur les relations négatives existant entre symptomatologie anxieuse et niveaux de conscience émotionnelle globale (CEG), intra- (CE-Intra) et inter- subjectives (CE-Inter) n'a pas été confirmée. Les résultats montrent que 
les symptômes anxieux sont corrélés positivement et de manière significative à la conscience émotionnelle. Des différences significatives sur différentes composantes du fonctionnement émotionnel en fonction de l'âge des individus faisaient l'objet d'une troisième hypothèse qui a été confirmée. Les résultats vont dans le sens d'une meilleure capacité de représentation de l'expressivité émotionnelle pour les individus les plus jeunes par rapport aux individus plus âgés, ainsi que de meilleures capacités de différenciation et de description de leurs états émotionnels. Enfin, notre dernière hypothèse postulait l'existence de liens entre différentes dimensions sous-jacentes aux échelles utilisées, donnant lieu à des interprétations en termes de profils de fonctionnement émotionnel. Cette hypothèse est confirmée par les analyses statistiques (analyse factorielle en composantes principales) qui montrent en effet l'existence de trois axes factoriels pouvant expliquer de manière significative la variance totale des scores des participants aux quatre échelles (HAD-AIM-EES-LEAS).

Au regard de l'ensemble de ces résultats, on pourrait considérer que le niveau de conscience émotionnelle joue un rôle dans l'étiologie des symptomatologies anxieuse et dépressive. Dans la littérature, un faible niveau de conscience émotionnelle correspondrait à un trait de personnalité alexithymique (alexithymie «primaire ») [28]. Ceci expliquerait qu'un sujet anxieux puisse présenter des tendances à l'intensité émotionnelle et la représentation d'une forte expressivité émotionnelle.

Pourtant, les résultats montrent que plus les individus souffrent de symptômes anxieux, plus ils présentent des capacités de différenciation et de description de leurs propres états émotionnels. Une récente recherche a obtenu des données similaires aux nôtres en comparant des individus présentant un 
trouble d'anxiété généralisée à une population contrôle [23]. Les individus anxieux se situaient à un niveau de CE-Intra supérieur aux individus non anxieux. Les auteurs posent la question de l'évaluation des capacités verbales (richesse du vocabulaire) chez les anxieux plutôt que de différenciation des états émotionnels, avec l'utilisation de la LEAS. En effet, d'autres études soulignent l'existence d'une corrélation significative entre la LEAS et la souséchelle «vocabulaire » de la WAIS-R ${ }^{1}$ [17] [9] cités dans [6]. Cependant, ceci ne semble pas être en opposition avec l'idée selon laquelle les individus anxieux seraient au niveau conceptuel, plus compétents dans l'identification et la description de leurs propres états émotionnels, dans la mesure où ils ont potentiellement une bonne pratique de l'analyse cognitive de leur émotions afin d'éviter les situations où ils pourraient les ressentir. Nous partageons le point de vue de Novick-Kline et collaborateurs [23] concernant l'aspect trop imaginatif des situations de vie proposées dans la LEAS. Il s'agit de situations imaginaires où il est demandé de répondre à priori sur les émotions que l'individu ressentirait. Les relations significatives entre symptomatologie anxieuse et conscience émotionnelle intra-subjective montrent que les capacités de différenciation et de description des émotions sont potentiellement présentes chez ces individus mais qu'elles ne sont sans doute pas exploitées. Une dimension émotionnelle serait peut-être à l'œuvre dans ce fonctionnement spécifique, c'est ce que d'autres travaux portent à croire. Une relation entre la dimension évaluant la symptomatologie anxieuse (HAD) et la dimension évaluant des difficultés d'identification des émotions (dimension de l'alextihymie évaluée par TAS-20²) a été établie par analyse factorielle [13].

\footnotetext{
${ }^{1}$ Weschler Adult Intelligence Scale Revised

2 Tonroto Alexithymia Scale (20 items)
} 
D'après les auteurs, la symptomatologie anxieuse semble partager des caractéristiques cognitives communes avec les difficultés d'identification des émotions. La nature de ce lien et des dimensions pouvant «moduler» ce lien n'est pas encore déterminée. A l'inverse, d'autres résultats montrent que les difficultés à identifier et décrire ses émotions apparaissent comme la conséquence (principalement cognitive) de l'humeur dépressive. Elles feraient donc partie inhérente de l'émoussement affectif [14]. Ces données vont dans le sens de nos résultats concernant l'absence de corrélation entre les symptômes dépressifs et le niveau de conscience émotionnelle. En effet, dans l'analyse des symptômes dépressifs, on ne retrouve pas de relation avec une conscience émotionnelle de niveau haut, comme c'est le cas dans l'anxiété. L'analyse factorielle (issue de ces corrélations) nous fournit davantage d'éléments de compréhension concernant les liens qui unissent les symptomatologies au niveau de conscience émotionnelle. On a vu que les dimensions sous-jacentes aux échelles pouvaient être réparties de trois manières différentes sur des axes factoriels cherchant à expliquer la variance totale des scores obtenus à l'ensemble des échelles utilisées. De ces trois axes factoriels interprétés comme la «connaissance de soi », la «différenciation des états émotionnels » et l'«abrasion émotionnelle » se dessinent trois grands profils de fonctionnement émotionnel :

- Des individus SA (qui présentent des symptômes anxieux) qui manifestent une «forte intensité et expressivité émotionnelle» sous-tendue par une capacité d'identification et description de ses émotions propres. Les SA parviendraient à s'imaginer et se représenter leurs émotions dans situations 
virtuelles mais pas concrètes (en lien avec leurs capacités d'anticipation cognitive pour éviter les situations à fort potentiel anxiogène) ;

- Des individus NSA (qui ne présentent pas de symptômes anxieux) qui ne perçoivent pas leur vécu émotionnel avec intensité et ne se considèrent pas comme fortement expressif sur le plan émotionnel, ont des capacités de différenciation et de description fines des états émotionnels. On pourrait alors penser que les capacités de différenciation des états émotionnels plus complexes sont possibles une fois la perception de l'intensité émotionnelle levée et les symptômes anxieux absents. Et ceci serait d'autant plus vrai pour les individus les plus jeunes (entre 20 et 40 ans).

- Enfin, des individus SA/SD (présence des symptômes anxieux et dépressifs) qui manifestent une abrasion des émotions positives et des représentations de soi dans le sens d'une absence de l'expressivité émotionnelle. La perception d'un vécu émotionnel négatif intense et d'une forte réactivité négative dominent. Les individus ne présentent pas de capacités de différenciations des états émotionnels spécifiques contrairement aux individus «symptômes anxieux » seulement (cf. premier profil).

Ces résultats doivent néanmoins être considérés avec prudence du fait des limites inhérentes à la constitution de l'échantillon. En effet, les sujets sont exclusivement féminins et le nombre est relativement limité. Les évaluations faites dans cette étude mériteraient d'être répliquées sur un échantillon d'hommes et de femmes, et de plus grande taille afin de repérer les variations dues au sexe. Des études montrent l'effet du sexe sur des variables telles que la réactivité émotionnelle. Les femmes auraient tendance à davantage exprimer les émotions négatives et à réagir à des stimuli négatifs par des émotions telles 
que l'anxiété et par des affects dépressifs, alors que les hommes seraient plus enclins à garder leurs émotions pour eux et à exprimer préférentiellement la colère [4]. Nous sommes ainsi amenés à rester réservés quant à une généralisation de nos résultats. Une autre limite due à la constitution de notre échantillon réside dans les niveaux d'intensité modérée des symptomatologies anxieuse et dépressive. Des symptomatologies franchement pathologiques auraient sans doute donné lieu à des résultats différents. Cependant, nos résultats sont concordants avec d'autres recherches menées auprès de la population générale où les résultats obtenus sont similaires à ceux issus des populations cliniques [13]. On peut penser que l'intensité des symptomatologies renforce les relations qu'elles entretiennent avec les dimensions émotionnelles sans changer la nature des liens.

Cette recherche pourrait permettre de sortir de cette dualité entre une vision de l'alexithymie-trait versus l'alexithymie-état. En effet, nous pensons, comme d'autres auteurs, qu'il n'existe pas de différence entre ces deux formes d'alexithymie dans la mesure où les deux renvoient à la même difficulté à utiliser la cognition pour identifier les émotions et en faire usage dans les interactions avec l'environnement [8]. Dans ce sens, des travaux montrent que l'alexithymie pourrait être considérée comme un facteur modérateur entre des conduites à risque et des symptômes dépressifs [2]. Selon nous, indépendamment du niveau de développement de la conscience émotionnelle des individus, c'est la présence de symptômes anxieux et/ou dépressifs qui, dans leur articulation avec les dimensions structurales de l'expérience émotionnelle, vont engendrer ou non une alexithymie secondaire comme un épiphénomène de l'état émotionnel de l'individu. L'appréhension de la 
dimension d'activation de l'émotion semble être cruciale dans la compréhension des capacités de différenciation des états émotionnels des individus anxieux. Pour les individus qui présentent des symptômes dépressifs ce serait alors la tendance à la valence négative de l'émotion qui serait plus déterminante (sensibilité à l'intensité émotionnelle versus valence négative).

Enfin, cette étude apporte des éléments importants pour la prise en charge des individus souffrant de symptômes anxieux. En effet, un travail sur la perception de l'individu quant à l'intensité du vécu émotionnel pourrait participer à l'amélioration de ses capacités de différenciation et de description des émotions. 


\section{Bibliographie}

[1] Barczack, P., Kane, N., Andrews, S., Congdon, A.M., Clay, J.C., \& Betts, T. Patterns of psychiatric morbidity in a genito-urinary clinic: a validation of the Hospital Anxiety Depression scale (HAD). Brit. J. Psychiatry 1988 ; 152, 698-700.

[2] Bréjard, V., Bonnet, A. et Pedinielli, J-L. Régulation des émotions, dépression et conduites à risques : l'alexithymie comme facteur modérateur, AMEPSY $2005 ; 613: 1-9$.

[3] Brochier, G. Coexistence des troubles anxieux et dépressifs. In Rapport itinéraire des déprimés. Ag Nat pour l'Ev Méd. 2001 ; 1995.

[4] Brody, L.R., Hall, J.A. Gender and emotion. In M. Lewis \& J.Haviland (Eds.), Handbook of emotions 1993. New-York: Guilford.

[5] Bryant, F.B., Yarnold, P.R., Grimm, L.G. Toward a measurement model of the affect intensity measure: A three factor structure. Jour of res in perso 1996; 30: 223-47.

[6] Ciarrochi, J., Scott, G. The link between emotional competence and wellbeing : a longitudinal study. Br J of Guid and Consel 2006; (34) 2: 231-43.

[7] Cogneau, J., Liard, F., Charbonnel, L. Prescription de psychotropes et prévalence des états dépressifs et(ou) anxieux en médecine générale. Rev Prat Med Gen $2003 ; 17$ : 1185-8.

[8] Corcos, M., Speranza, M. Psychopathologie de l'alexithymie. Paris : Dunod ; 2003.

[9] Croyle, K.L., Waltz, J. Emotional awareness and couples' relationship satisfaction. J of Marit and Fam Ther 2002; 28: 435-44.

[10] Feldman, L.A. Variations in the circumplex structure of mood. Pers and Soc Psych Bul 1995a; 21: 806-17.

[11] Feldman, L.A. Valence-focus and arousal-focus: Individual differences in the structure of affective differences. J of Pers and Soc Psych Bul 1995b; 69: 153-66.

[12] Hintikka, J., Honkalampi, K., Lehtonen, J., Viinamaki, H. Are alexithymia and depression distinct or overlapping construct?: A study in a general population. Compr Psychiatry 2001; 42:234-9.

[13] Honkalampi K., Hintikka, J., Tanskanen, A., Lehtonen, J., Viinamaki, H. Depression is strongly associated with alexithymia in the general population. $\mathrm{J}$ Psychosom Res 1999; 48:99-104.

[14] Kring, A. M., Smith, D. A., Neale, J. M. Individual differences in the dispositional expressiveness : developpement and validation of the emotional Expressivity Scale. J. Pers. Soc. Psychol. 1994 ; 66 : 934-949.

[15] Lane, RD., Quinlan, D.M., Schwartz, GE., Walker, PA., Zeitlin, SB. The levels of emotional awareness scale: a cognitive-developmental measure of emotion. J of Pers Asses 1990; 55(1\&2), 124-134.

[16] Lane, R.D., Schwartz, G.E. Levels of emotional awareness: a cognitvedevelopmental theory and ist application to psychopathology. Amer $\mathbf{J}$ of Psych1987; 144: 133-43.

[17] Lane, R.D., Sechrest, L., Riedel, R. Sociodemographic correlates of alexithymia. Compr Psych 1998; 39: 277-85.

[18] Lane, R.D., Sechrest, L., Riedel, R., Shapiro, D.E., Kaszniak, A.W. Pervasive emotion recognition deficit common to alexithymia and the repressive coping style. Psychosom Med 2000; 62: 492-501. 
[19] Larsen, R.J. Theory and measurement of affect intensity as an individual differences charactestic. Diss Abst Intern 1984; 85: 2297B.

[20] Larsen, R.J., Diener, E. Affect intensity as an individual difference characteristic: A review. J of Res in Pers 1987; 21: 1-39.

[21] Lépine, J.P., Godchau, M., Brun, P., Lemperiere, T. Evaluation de l'anxiété et de la dépression chez des patients hospitalisés dans une service de médecine interne. Ann Med-psychol 1985 ; 143 : 175-189.

[22] Marchesi, C., Brusamonti, E., Maggini, C. Are alexithymia, depression and anxiety distinct constructs in affective disorders ? J of Psychosom Res $2000 ; 49$ : 43-49.

[23] Novick-Kline, P., Turk, C.L., Mennin, D.S., Hoyt, E.A., Gallagher, C.L. Level of emotional awareness as a differentiating variable between individuals with and without generalized anxiety disorder. Anx Dis 2004; 19(2005): 55772.

[24] Pedinielli, J-L. et Bernoussi, A. Les états dépressifs. Paris : Nathan; 2004.

[25] Pichot, P. Les dépressions : problèmes de vocabulaire et nosologie. Les voies nouvelles de la dépression. Paris : Masson ; 1978.

[26] Russel, J.A., Caroll, J.M. On the bipolarity of positive and negative affect. Psychol Bul 1999a; 125: 3-30.

[27] Sifnéos, PE. The prevalence of alexithymic characteristics in psychosomatic patients. Psychother Psychosom 1973; 22:255-62.

[28] Taylor, G.J., Bagby, R.M., Parker, J.D.A. Disorders of affect regulation. Alexithymia in medical and psychiatric illness. Cambridge: Cambridge and Univerversity Press; 1997.

[29] Taylor, L., Gorman, J. Theoritical and therapeutic considerations for anxiety disorders. Psych Quaterly 1992; 63(4): 319-42.

[30] Watson, D., Clark, L.A. Negative affectivity : the disposition to experience unpleasant emotional states. Psyc Bul 1984; 95: 465-90.

[31] Watson, D., Tellegen, A. Issues in the dimensional structure of affectEffects of descriptors, measurement error, and response formats: Comment on Russell and Carroll (1999). Psychol BUl 1999; 125: 609-10.

[32] Zigmond, A.S., \& Snaith, R.P. The Hospital Anxiety and Depression Scale. Acta Psychiatr. Scand, 1983, 67, 361-370. 
Tableau 1 :

Effectif total $(\mathrm{N}=50)$ : statistiques descriptives pour les mesures des composantes émotionnelles et cognitives

\begin{tabular}{|r|l|r|r|r|r|r}
\hline Questionnaires & Variables mesurées & \multicolumn{1}{l}{ Moyenne } & Ec. Type & Médiane & Min & Max \\
\hline \multirow{2}{*}{ HAD } & Symptômes anxieux & 7,92 & 2,69 & 7,50 & 2 & 15 \\
\cline { 2 - 7 } & Symptômes dépressifs & 2,86 & 1,63 & 3,00 & 0 & 7 \\
\hline \multirow{3}{*}{ AIM } & Intensité émotionnelle & 143,54 & 20,97 & 144,50 & 102 & 178 \\
\cline { 2 - 7 } & Affectivité positive & 56,82 & 12,10 & 55,00 & 35 & 79 \\
\cline { 2 - 7 } & Intensité négative & 20,76 & 5,43 & 21,00 & 10 & 34 \\
\cline { 2 - 7 } & Réactivité négative & 26,26 & 5,46 & 27,00 & 13 & 34 \\
\hline \multirow{2}{*}{ EES } & Expressivité & 68,52 & 12,64 & 69,00 & 45 & 94 \\
\hline \multirow{3}{*}{ NCE } & Conscience émotionnelle & 58,04 & 8,44 & 59,00 & 36 & 77 \\
\cline { 2 - 7 } & CE Intra-subjective & 51,88 & 8,27 & 54,00 & 30 & 67 \\
\cline { 2 - 7 } & CE Inter-subjective & 47,50 & 8,45 & 47,50 & 27 & 65 \\
\hline
\end{tabular}


Tableau 2 :

Corrélations entre l'âge, les symptomatologies anxieuse, dépressive et les dimensions émotionnelles et le niveau de développement cognitivo-émotionnel (effectif total $\mathrm{N}=50$ )

\begin{tabular}{|c|c|c|c|c|c|c|c|c|c|c|}
\hline & (2) & (3) & (4) & (5) & (6) & (7) & (8) & (9) & (10) & (11) \\
\hline Age (1) & $-0,02$ & $0,25^{\star \star \star}$ & $-0,07$ & 0,04 & $0,14^{\star}$ & 0,10 & $-0,26^{\star \star \star}$ & $-0,38^{\star \star}$ & $-0,37^{\star \star \star}$ & $-0,28^{\star \star \star}$ \\
\hline Symptômes anxieux (2) & & $0,45^{\star \star \star}$ & $0,30^{\star \star \star}$ & $0,23^{\star \star \star}$ & $0,42^{\star \star \star}$ & $0,38^{\star \star \star}$ & $-0,12$ & $0,31^{\text {** }}$ & $0,32^{\star \star \star}$ & $0,25^{\star \star \star}$ \\
\hline Symptômes dépressifs (3) & & & 0,05 & $-0,12$ & $0,35^{\star \star \star}$ & $0,34^{\star \star \star}$ & $-0,31^{\star \star \star}$ & 0,03 & 0,08 & $-0,07$ \\
\hline Intensité émotionnelle (4) & & & & $0,87^{\star \star \star}$ & $0,69^{\star \star \star}$ & $0,61^{\star \star \star}$ & $0,52^{\star \star \star}$ & $0,20^{\star \star \star}$ & $0,25^{\star \star \star}$ & 0,09 \\
\hline Affectivité positive (5) & & & & & $0,59^{\star \star \star}$ & $0,50^{\star \star \star}$ & $0,41^{\star \star \star}$ & $0,17^{\star \star}$ & $0,21^{\star *}$ & $0,16^{\star \star}$ \\
\hline Intensité négative (6) & & & & & & $0,72^{\star \star \star}$ & $0,25^{\star \star \star}$ & 0,10 & $0,19^{\star \star}$ & $-0,01$ \\
\hline Réactivité négative (7) & & & & & & & $0,24^{\star \star \star}$ & $0,21^{\star \star \star}$ & $0,21^{\star \star}$ & 0,11 \\
\hline Expressivité émotionnelle (8) & & & & & & & & $-0,01$ & $-0,02$ & $-0,09$ \\
\hline Conscience émotionnelle (9) & & & & & & & & & $0,92^{\star \star \star}$ & $0,87^{\star \star \star}$ \\
\hline NCE intrasubjective (10) & & & & & & & & & & $0,71^{\star \star \star}$ \\
\hline NCE intersubjective (11) & & & & & & & & & & 1,00 \\
\hline
\end{tabular}


Diagramme 1: Comparaisons de moyennes aux différentes dimensions cognitivoémotionnelles selon le groupe d'âge pour l'ensemble des sujets $(\mathrm{N}=50)$.

« $\mathrm{S} »=$ différences de moyennes significatives au seuil $\mathrm{p}<.05$.

Comparaisons de moyennes aux dimensions émotionnelles et niveau de développement cognitivo-émotionnel selon le groupe d'âge

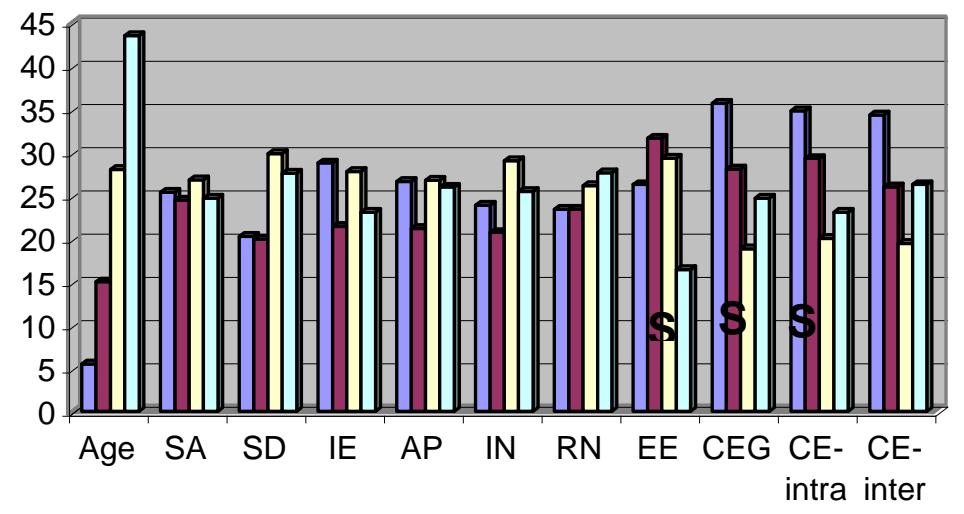


Tableau 3 :

Poids factoriel par variable (a), valeurs propres (b) et pourcentages de variances expliquées (c) par les axes factoriels avant rotation (effectif total $\mathrm{N}=50$ ).

\begin{tabular}{|l|r|r|r|}
\hline & Facteur 1 (a) & Facteur 2 (a) & Facteur 3 (a) \\
\hline Age & $-0,18$ & $\mathbf{- 0 , 5 0}$ & 0,45 \\
\hline Symptômes anxieux & $\mathbf{0 , 5 6}$ & 0,02 & $\mathbf{0 , 5 2}$ \\
\hline Symptômes dépressifs & 0,21 & $-0,18$ & $\mathbf{0 , 8 1}$ \\
\hline Intensité émotionnelle & $\mathbf{0 , 8 1}$ & $-0,41$ & $-0,28$ \\
\hline Affectivité positive & $\mathbf{0 , 7 3}$ & $-0,37$ & $-0,33$ \\
\hline Intensité négative & $\mathbf{0 , 7 3}$ & $-0,49$ & 0,17 \\
\hline Réactivité négative & $\mathbf{0 , 7 3}$ & $-0,37$ & 0,18 \\
\hline Expressivité & $\mathbf{0 , 3 3}$ & $-0,31$ & $\mathbf{- 0 , 7 1}$ \\
\hline Conscience émotionnelle & $\mathbf{0 , 6 4}$ & $\mathbf{0 , 7 4}$ & 0,03 \\
\hline NCE intrasubjectif & $\mathbf{0 , 6 5}$ & $\mathbf{0 , 6 5}$ & 0,05 \\
\hline NCE intersubjectif & 0,50 & $\mathbf{0 , 7 4}$ & 0,01 \\
\hline Valeurs propres (b) & 3,82 & 2,57 & 1,88 \\
\hline \% variance expliquée (c) & 0,35 & 0,23 & 0,17 \\
\hline
\end{tabular}

